DEMONSTRATIO MATHEMATICA

$\begin{array}{lll}\text { Vol. XXX } & \text { No } 1 \quad 1997\end{array}$

Dinh Van Ruy, Nguyen Thanh Long, Duong Thi Thanh Binh

\title{
ON A NONEXISTENCE OF POSITIVE SOLUTION OF LAPLACE EQUATION IN UPPER HALF-SPACE
}

\section{Introduction}

Consider the following Laplace equation in the upper half-space

$$
\Delta u=0, \quad(x, y, z) \in R_{+}^{3}=\left\{(x, y, z) \in R^{3}: z>0\right\}
$$

with a nonlinear boundary condition of the form

$$
-u_{z}(x, y, 0)=f(x, y, u(x, y, 0)), \quad(x, y) \in R^{2} .
$$

In [1] there was studied the Laplace equation of axial symmetry form

$$
u_{r r}+\frac{1}{r} u_{r}+u_{z z}=0, \quad r>0, z>0,
$$

with a nonlinear boundary condition

$$
-u_{z}(r, 0)=I_{0} \exp \left(-r^{2} / r_{0}^{2}\right)+u^{\alpha}(r, 0), \quad r \geq 0,
$$

where $I_{0}, r_{0}, \alpha$ are given positive constants. The problem (1.3), (1.4) is a stationary case of the problem relative to ignition by radiation. In [1] it was proved that the problem (1.3), (1.4) in the case $0<\alpha \leq 2$ has no positive solution. Afterwards, this result has been extended in [2] for more general nonlinear boundary condition

$$
-u_{z}(r, 0)=g(r, u(r, 0)), r \geq 0 .
$$

In this paper we consider the problem (1.1), (1.2) with a given function $f$ which is continuous, nondecreasing and bounded below by the power function of order $\alpha$ with respect to the third variable. By constructing a suitable functional sequence, we prove that for $0<\alpha \leq 2$ the problem (1.1), (1.2) has no positive solution. This result is a relative extension of that from [1], [2]. 
2. Hypotheses and statement of the main result

We omit the definition of a usual function space. Put

$$
R_{+}=\{x \in R: x \geq 0\} .
$$

Assume that the function $f: R^{2} \times R_{+} \rightarrow R$ satisfies the following conditions:

$\left(\mathrm{H}_{1}\right) f$ is continuous,

$\left(\mathrm{H}_{2}\right) f$ is nondecreasing with respect to third variable, i.e.,

$$
(f(x, y, u)-f(x, y, v))(u-v) \geq 0, \quad \forall x, y \in R, \quad \forall u, v \in R_{+},
$$

$\left(\mathrm{H}_{3}\right)$ there exist two positive constants $\alpha$ and $M$ such that

$$
f(x, y, u) \geq M u^{\alpha}, \quad \forall x, y \in R, \quad \forall u \in R_{+},
$$

$\left(\mathrm{H}_{4}\right)$ the intergal $\iint_{R^{2}} \frac{f(x, y, 0) d x d y}{1+\sqrt{x^{2}+y^{2}}}$ exists and is positive.

Besides, the solution of the problem (1.1), (1.2) is supposed to satisfy the following conditions:

$\left(\mathrm{S}_{1}\right) u \in C^{2}\left(R_{+}^{3}\right) \cap C\left(\overline{R_{+}^{3}}\right), u_{z} \in C\left(\overline{R_{+}^{3}}\right)$, where

$$
\overline{R_{+}^{3}}=\left\{(x, y, z) \in R^{3}: z \geq 0\right\},
$$

$\left(\mathrm{S}_{2}\right) u$ is regular at infinity.,i.e.,

(i) $\max _{x^{2}+y^{2}+z^{2}=R^{2}}|u(x, y, z)| \rightarrow 0$, as $R \rightarrow+\infty$,

(ii) there exists a constant $C>0$ such that

$$
|\operatorname{grad} u(x, y, z)| \leq \frac{C}{x^{2}+y^{2}+z^{2}}
$$

as $x^{2}+y^{2}+z^{2}$ is sufficiently large.

The solution $u$ of the problem (1.1), (1.2) satisfying the conditions $\left(S_{1}\right)$, $\left(\mathrm{S}_{2}\right)$ can be represented by (see [3])

$$
u(x, y, z)=A[f(\xi, \eta, u(\xi, \eta, 0))](x, y, z), \quad \forall(x, y, z) \in R_{+}^{3},
$$

where $A$ is the linear operator defined by

$$
A[v(\xi, \eta)](x, y, z)=\frac{1}{2 \pi} \iint_{R^{2}} \frac{v(\xi, \eta) d \xi d \eta}{\sqrt{(x-\xi)^{2}+(y-\eta)^{2}+z^{2}}} .
$$

We state the main result of this paper as follows.

THEOREM 1. Suppose that $f$ satisfies the hypotheses $\left(\mathrm{H}_{1}\right)-\left(\mathrm{H}_{4}\right)$ with $0<\alpha \leq 2$. Then the problem (1.1), (1.2) has no positive solution satisfying $\left(\mathrm{S}_{1}\right),\left(\mathrm{S}_{2}\right)$. 


\section{Proof of Theorem 1}

Suppose, by contradiction, that the problem (1.1), (1.2) has a positive solution $u(x, y, z)$ satisfying $\left(\mathrm{S}_{1}\right),\left(\mathrm{S}_{2}\right)$. Let $z \rightarrow 0_{+}$in the integral equation (2.1) and put $u(x, y, 0)=u(x, y)$. Then we obtain

$$
u(x, y)=A[f(\xi, \eta, u(\xi, \eta))](x, y),
$$

where $A$ is a linear operator defined by

$$
A[v(\xi, \eta)](x, y)=\frac{1}{2 \pi} \iint_{R^{2}} \frac{v(\xi, \eta) d \xi d \eta}{\sqrt{(x-\xi)^{2}+(y-\eta)^{2}}} .
$$

Construct a recurrent functional sequence $\left\{u_{n}(x, y)\right\}$ defined by

$$
\begin{gathered}
u_{1}(x, y)=\frac{m_{1}}{1+\sqrt{x^{2}+y^{2}}}, \quad m_{1}=\frac{1}{2 \pi} \iint_{R^{2}} \frac{f(\xi, \eta, 0) d \xi d \eta}{1+\sqrt{\xi^{2}+\eta^{2}}} \\
u_{n+1}(x, y)=A\left[f\left(\xi, \eta, u_{n}(\xi, \eta)\right)\right](x, y), \quad n \geq 1 .
\end{gathered}
$$

Then, we have two following lemmas.

LEMMA 1.

$$
A[f(\xi, \eta, 0))](x, y) \geq u_{1}(x, y), \quad \forall x, y \in R .
$$

P r o of. From the inequalities

$$
\begin{aligned}
& \frac{1}{\sqrt{(x-\xi)^{2}+(y-\eta)^{2}}} \geq \frac{1}{\sqrt{x^{2}+y^{2}}+\sqrt{\xi^{2}+\eta^{2}}} \\
& \geq \frac{1}{1+\sqrt{x^{2}+y^{2}}} \cdot \frac{1}{1+\sqrt{\xi^{2}+\eta^{2}}}, \\
& \forall x, y, \xi, \eta \in R,
\end{aligned}
$$

by (3.3), we obtain (3.5).

LEMMA 2. The functional sequence $\left\{u_{n}(x, y)\right\}$ defined by (3.3), (3.4) satisfies the following conditions:

(i) $\left\{u_{n}\right\}$ is nondecreasing.,i,e.,

$$
u_{n}(x, y) \leq u_{n+1}(x, y), \quad \forall n \in N, \quad \forall x, y \in R .
$$

(ii) $\left\{u_{n}\right\}$ is bounded above by $u(x, y)$.,i.e.,

$$
u_{n}(x, y) \leq u(x, y), \quad \forall n \in N, \quad \forall x, y \in R .
$$

(iii) $u_{n}(x, y)$ converges pointwise and satisfies

$$
\lim _{n \rightarrow+\infty} u_{n}(x, y) \leq u(x, y), \quad \forall x, y \in R .
$$


P r o of. Runs easily by recurrence on $n$.

By means of (3.8), (3.9), the Theorem 1 will be proved, if we can show that

(a) there exists $n \in N$ such that $u_{n}(x, y)=+\infty, \quad \forall x, y \in R$, or

(b) there exists $(x, y)$ such that $\lim _{n \rightarrow+\infty} u_{n}(x, y)=+\infty$.

Further, we consider three cases of different values of $\alpha$. (see $\left(\mathrm{H}_{3}\right)$ ).

Case $1.0<\alpha \leq 1$.

Lemma 3. For $0<\alpha \leq 1$ we have

$$
A\left[\left(1+\sqrt{\xi^{2}+\eta^{2}}\right)^{-\alpha}\right](x, y)=+\infty, \quad \forall x, y \in R .
$$

P roof. Using the first inequality in (3.6) and then changing to polar coordinates, we obtain

$$
\begin{aligned}
A\left[\left(1+\sqrt{\xi^{2}+\eta^{2}}\right)^{-\alpha}\right](x, y) \\
\quad \geq \frac{1}{2 \pi} \iint_{R^{2}} \frac{d \xi d \eta}{\left(1+\sqrt{\xi^{2}+\eta^{2}}\right)^{\alpha}\left(\sqrt{\xi^{2}+\eta^{2}}+\sqrt{x^{2}+y^{2}}\right)} \\
\quad=\int_{0}^{+\infty} \frac{r d r}{(1+r)^{\alpha}\left(r+\sqrt{x^{2}+y^{2}}\right)} \\
\quad=+\infty .
\end{aligned}
$$

From Lemma 3 and the hypothesis $\left(\mathrm{H}_{3}\right)$ we have

$$
u_{2}(x, y) \geq M m_{1}^{\alpha} A\left[\left(1+\sqrt{\xi^{2}+\eta^{2}}\right)^{-\alpha}\right](x, y)=+\infty .
$$

Hence, the Theorem 1 is proved in Case 1.

Case 2. $1<\alpha<2$.

Lemma 4. For $\alpha>1$ we have

$$
A\left[\left(1+\sqrt{\xi^{2}+\eta^{2}}\right)^{-\alpha}\right](x, y) \geq \frac{1}{2(\alpha-1)\left(1+\sqrt{x^{2}+y^{2}}\right)^{\alpha-1}} .
$$

P roof. Similarly as in (3.11) we have

$$
A\left[\left(1+\sqrt{\xi^{2}+\eta^{2}}\right)^{-\alpha}\right](x, y) \geq \int_{\sqrt{x^{2}+y^{2}}}^{+\infty} \frac{r d r}{(1+r)^{\alpha}\left(r+\sqrt{x^{2}+y^{2}}\right)}
$$


From the inequality

$$
\frac{r}{r+\sqrt{x^{2}+y^{2}}} \geq \frac{1}{2}, \quad \forall r \geq \sqrt{x^{2}+y^{2}}
$$

we obtain (3.12) and Lemma 4 is proved.

Using now the recurrent relation $(3.3),(3.4)$, hypothesis $\left(\mathrm{H}_{3}\right)$ and Lemma 4 , we obtain

$$
u_{2}(x, y) \geq A\left[M u_{1}^{\alpha}(\xi, \eta)\right](x, y) \geq m_{2}\left(1+\sqrt{x^{2}+y^{2}}\right)^{-\lambda_{2}}
$$

where

$$
\lambda_{2}=\alpha-1, \quad m_{2}=M m_{1}^{\alpha} / 2 \lambda_{2}
$$

By recurrence, we can prove that

$$
u_{n}(x, y) \geq m_{n}\left(1+\sqrt{x^{2}+y^{2}}\right)^{-\lambda_{n}}
$$

with

$$
\lambda_{n}=\frac{1-(2-\alpha) \alpha^{n-1}}{\alpha-1}, \quad m_{n}=\frac{M m_{n-1}^{\alpha}}{2 \lambda_{n}}, \quad \forall n \geq 2 .
$$

Since $1<\alpha<2$, we can choose a natural number $N$ (depending on $\alpha$ ), such that

$$
\frac{-\ln (2-\alpha)}{\ln \alpha} \leq N<1-\frac{\ln (2-\alpha)}{\ln \alpha},
$$

namely we choose $N$ such that $0<\alpha \lambda_{N} \leq 1$. By hypothesis $\left(\mathrm{H}_{3}\right)$ and Lemma 3, we have

$$
u_{N+1}(x, y) \geq M m_{N}^{\alpha} A\left[\left(1+\sqrt{\xi^{2}+\eta^{2}}\right)^{-\alpha \lambda_{N}}\right](x, y)=+\infty .
$$

Therefore, Theorem 1 is proved in Case 2.

Case 3. $\alpha=2$.

LEMMA 5. We have

$$
A\left[\left(1+\sqrt{\xi^{2}+\eta^{2}}\right)^{-2}\right](x, y) \geq \frac{\ln \left(1+\sqrt{x^{2}+y^{2}}\right)}{4 \sqrt{x^{2}+y^{2}}} .
$$

P r o of. Similarly as in (3.11) we have

$$
A\left[\left(1+\sqrt{\xi^{2}+\eta^{2}}\right)^{-2}\right](x, y) \geq \int_{1}^{+\infty} \frac{r d r}{(1+r)^{2}\left(r+\sqrt{x^{2}+y^{2}}\right)}
$$


Using the inequality

$$
\frac{r}{(1+r)^{2}} \geq \frac{1}{4 r}, \quad \forall r \geq 1
$$

we have (3.20).

Lemma 6. Putting

$$
v_{n}(x, y)= \begin{cases}0, & x^{2}+y^{2} \leq 1, \\ \frac{C_{n}}{\sqrt{x^{2}+y^{2}}}\left(\ln \sqrt{x^{2}+y^{2}}\right)^{2^{n-2}}, & x^{2}+y^{2} \geq 1,\end{cases}
$$

where

$$
C_{n}=M^{2^{n-1}-1}\left(\frac{1}{2} m_{1} \sqrt{\ln 2}\right)^{2^{n-1}} \frac{1}{\ln 2}
$$

we have

$$
u_{n}(x, y) \geq v_{n}(x, y), \quad \forall x, y \in R, \quad \forall n \geq 2 .
$$

P r o of. First, observe that the inequality (3.25) holds for $n=2$. Indeed, using Lemma 5 , we have

$$
\begin{aligned}
u_{2}(x, y) & \geq M m_{1}^{2} A\left[\left(1+\sqrt{\xi^{2}+\eta^{2}}\right)^{-2}\right](x, y) \\
& \geq \frac{C_{2}}{\sqrt{x^{2}+y^{2}}} \ln \left(1+\sqrt{x^{2}+y^{2}}\right) .
\end{aligned}
$$

Hence, from (3.23), (3.26) we have

$$
u_{2}(x, y) \geq v_{2}(x, y), \quad \forall x, y \in R .
$$

Suppose that (3.25) holds for a fixed $n$. It is easy to see that

$$
u_{n+1}(x, y) \geq 0, \quad \forall x, y \in R .
$$

With $x^{2}+y^{2} \geq 1$, we have

$$
\begin{aligned}
u_{n+1}(x, y) & \geq M A\left[v_{n}^{2}(\xi, \eta)\right](x, y) \\
& \geq M C_{n}^{2} \int_{\sqrt{x^{2}+y^{2}}}^{+\infty} \frac{(\ln r)^{2^{n-1}} d r}{r\left(r+\sqrt{x^{2}+y^{2}}\right)} \\
& \geq M C_{n}^{2}\left(\ln \sqrt{x^{2}+y^{2}}\right)^{2^{n-1}} \int_{\sqrt{x^{2}+y^{2}}}^{+\infty} \frac{d r}{r\left(r+\sqrt{x^{2}+y^{2}}\right)}
\end{aligned}
$$




$$
\begin{aligned}
& =\frac{M C_{n}^{2} \ln 2}{\sqrt{x^{2}+y^{2}}}\left(\ln \sqrt{x^{2}+y^{2}}\right)^{2^{n-1}} \\
& =\frac{C_{n+1}}{\sqrt{x^{2}+y^{2}}}\left(\ln \sqrt{x^{2}+y^{2}}\right)^{2^{n-1}}, \quad \forall x, y \in R,
\end{aligned}
$$

because $M C_{n}^{2} \ln 2=C_{n+1}$, by (3.24).

From (3.23), (3.28) we obtain

$$
u_{n+1}(x, y) \geq v_{n+1}(x, y), \quad \forall x, y \in R .
$$

This fact shows by induction that Lemma 6 is true.

By (3.23), (3.24), we rewrite $v_{n}(x, y)$ for $x^{2}+y^{2} \geq 1$ in the form

$$
v_{n}(x, y)=\frac{1}{M \sqrt{x^{2}+y^{2}} \ln 2}\left(\frac{1}{2} M m_{1} \sqrt{\ln 2 \ln \sqrt{x^{2}+y^{2}}}\right)^{2^{n-1}} .
$$

Choose $x, y$ such that $\frac{1}{2} M m_{1} \sqrt{\ln 2 \ln \sqrt{x^{2}+y^{2}}}>1$, or

$$
x^{2}+y^{2}>\exp \left(8 / M^{2} m_{1}^{2} \ln 2\right)=r_{0}^{2} \text {. }
$$

Then we have

$$
\lim _{n \rightarrow+\infty} u_{n}(x, y) \geq \lim _{n \rightarrow+\infty} v_{n}(x, y)=+\infty, x^{2}+y^{2}>r_{0}^{2} .
$$

Hence Theorem 1 is proved in Case 3.

Combining Cases 1-3 we see that Theorem 1 holds for $0<\alpha \leq 2$.

Remarks: (i) In [1], the function $v_{n}(x, y)$ is given in the form of a functional series and is more complicated than (3.23).

(ii) The conclusion does not hold for $\alpha>2$. For example let $\alpha=3$ and $f(x, y, u)=k u^{3}$, where $k$ is a given positive constant. Of course, $f$ does not satisfy the hypothesis $\left(\mathrm{H}_{4}\right)$. The function $v(x, y, z)=\left(x^{2}+y^{2}+(z+k)^{2}\right)^{-1 / 2}$ is a positive solution of the problem (1.1), (1.2) satisfying $\left(\mathrm{S}_{1}\right),\left(\mathrm{S}_{2}\right)$.

Acknowledgement. The authors would like to thank the referee for his corrections and suggestions.

\section{References}

[1] F.V. Bunkin, V.A. Galaktionov, N.A. Kirichenko, S.P. Kurdyumov, A.A. Samarsky, On a nonlinear boundary value problem of ignition by radiation, J. Comp. Math. Phys. 28 (1988), 549-559. (in Russian).

[2] Nguyen Thanh Long, Dinh Van Ruy, On a nonexistence of positive solution of Laplace equation in upper half-space with Cauchy data, Demonstratio Math. 28 (1995), 921-927. 
[3] V.S. Vladimirov, Equations of Mathematical Physics, Mir Publishers Moscow, 1984.

Addresses of authors:

Dinh Van Ruy

COOPERATOR AT DEPARTMENT OF APPLIED MATHEMATICS, POLYTECHNIC UNIVERSITY OF HOCHIMINH CITY,

268 Ly Thuong Kiet str. dist. 10

HOCHIMINH CITY, VIETNAM

Nguyen Thanh Long

DEPARTMENT OF APPLIED MATHEMATICS

POLYTECHNIC UNIVERSITY OF HOCHIMINH CITY

268 Ly Thuong Kiet str. dist. 10

HOCHIMINH CITY, VIETNAM

Duong Thi Thanh Binh

DEPARTMENT OF PHYSICS-MATHEMATICS

UNIVERSITY OF MEDICINE AND PHARMACY OF HOCHIMINH CITY,

217 An Duong Vuong str. dist. 5

HOCHIMINH CITY, VIETNAM

Received April 14, 1995. 